\title{
Unified train data wireless transmission and management system for CRH series EMUs
}

\author{
Y. Sang ${ }^{1}$, J. $\operatorname{Han}^{2}$ \& H. Lü ${ }^{1}$ \\ ${ }^{1}$ Institute of Computing Technologies, \\ Chinese Academy of Railway Sciences, P. R. China \\ ${ }^{2}$ Passenger Car Department, China South Locomotive \& Rolling Stock \\ Corporation Limited, P.R. China
}

\begin{abstract}
CRH series Electric Multiple Units (EMUs) are equipped with a variety of advanced sensors and diagnostic units, which can generate real-time monitoring and diagnostic data representing the current technical states of their essential sub-systems. Timely acquiring and analyzing the monitoring and diagnostic data is significant for operation monitoring, emergency response and routine maintenance. To relieve the inconvenience of uploading and handling the data from different types of EMUs and to enhance the efficiency and effects of utilizing the dynamic data, the railway operation authority launched a project to work out an integrated solution and focus on formulating related technical specifications to build a unified train data wireless transmission and management system for CRH series EMUs. This paper addresses the requirements for such a unified system and summarizes the preliminary results of the project.

Keywords: train monitoring and diagnostic data, wireless data transmission, remote monitoring, management system, vehicle maintenance, standardization.
\end{abstract}

\section{Introduction}

Since June 17 2007, CRH series Electric Multiple Units, for short CRH EMUs and including CRH1, CRH2, CRH3, and CRH5 series, have been put into normal operation on some speed-raised existing railways and newly built passenger dedicated railways and intercity high-speed railways undergoing rapid expansion in China. These CRH EMUs are highly intelligent high speed trains installed with adavced Trian Control and Management Systems, which integrate 
major control subsytems and monitor devices aboard the EMUs and can transfer real-time controlling data, monitoring data and fault diagnositic data digitally via a train communication network to enable the concentrated control and monitor of various major functionalities of the EMUs. On the trains, the monitoring data and fault diagnositic data is displayed on the divers' monitors and on the Intelligent Display Units for other onboard crew, including traction, brakes, high voltage system, air supply, bogie, air conditioning and heating, doors, fire detection, passenger information system, etc.

Originally, all the CRH1 series, CRH3 series, and CRH5 series EMUs provide remote data transmission to automatically upload the monitoring data and fault diagnostic data to ground servers via GSM-based wireless transmission devices while the $\mathrm{CRH} 2$ series EMUs require manual data uploading by using a dedicated portable device. For each type of the CRH series EMUs, the frequency of data collection and the moment of data uploading were different, the uploaded data was stored in individual data files with unique formats, and one suit of dedicated software was installed to handle the uploaded data. Usually, one EMU depot or maintenance base should undertake the servicing and maintenance tasks for more than two different types of $\mathrm{CRH}$ series EMUs. So, it became a laborious routine for the depots and the maintenance bases to handle and manage the uploaded data. Otherwise, the differentiated definition of data categories, and the diversity of data content, and decentralized storage of the data made it too tough to utilize the data. Besides, due to the different design of the data uploading systems for each type of the CRH series EMUs, it was too hard to manage and maintain a stable operation of data uploading and data uploading is often interrupted due to various untold reasons.

Being convinced of the value and significance of the monitoring data and fault diagnostic data from the EMUs for emergency response and maintenance, the railway operation authority initiated a project to establish a unified train data wireless transmission and management system for CRH series EMUs at the beginning of the year 2009 and set up a working group. The workgroup first defined the requirements of the system and the categories of train data and formulated the Technical Specification of Train Data Wireless Transmission Devices for CRH Series EMU issued by the railway operation authority in May, 2010. It was required that the EMU manufacturers should design or redesign their onboard data remote transmission devices and conducted development or modification trials for each types of their EMUs, including the old types already in service and under manufacturing as well as the new types under design, i.e., later launched $\mathrm{CRH} 380 \mathrm{~A} / \mathrm{B} / \mathrm{C} / \mathrm{D}$, according to the specification. Meanwhile, a unified train data management system was set up for automatically receiving and handling the data in a centralized manner to provide access services for various users. 


\section{System requirements and definitions of train data}

\subsection{System requirements}

The workgroup investigated the needs of utilizing the train data among potential users from the servicing and maintenance fields, the traffic control centers and the EMU manufactures and discussed the feasibility of train data provision with all the EMU manufacturers. The major requirements of the unified train data wireless transmission and management system were concluded as follows:

- Major faults should be sent to the ground in a real-time manner to help the experts better support the driver and the onboard crew remotely in a case of emergency response.

$>$ The operating status of all the running EMUs can be monitored, tracked and traced collectively on the ground, facilitating the control centres to master the conditions of all the running EMUs in a integral view.

$>$ Train data should meet the needs of maintenance-oriented analysis and can be utilized to support timely fault repair, on-condition preventive maintenance, and conduct reliability monitoring.

The train data from all types of EMUs can be uploaded and stored in database for flexible and parallel access by various users by using one suit of common software, thus reducing the burden of the servicing and maintenance fields to handle it and meanwhile affording more secure and more reliable management of train data.

Newly installed onboard wireless transmission devices should be easy in operation management and maintenance for the sake of stable and reliable train data uploading, enabling status monitoring, self-diagnostics for transmission conditions, quick analysis of and recovery from faults, remote setting of configuration options, etc.

In consideration of the limits of real-time remote transmission, both mobile transmission and WLAN transmission will be combined in the new standard train data transmission device.

\subsection{Definitions of train data categories}

To meet the requirements above listed, six categories of train data are defined as follows:

$>$ Real-time GPS data - GPS data that include EMU number, train number, time, velocity, longitude, latitude, elevation, acceleration, etc., mainly for running position tracking. The real-time GPS data will be sampled at an interval of no more than 1 second and immediately sent via mobile transmission channel.

D Real-time fault data - major faults that could affect the normal operation of an EMU and their critical environment or context data, mainly for emergency response and remote expert support. The fault data includes fault code, failure time, fault location, velocity and driving/braking status when the fault occurs while its context data is the samplings of the parameters related to the 
fault during certain duration of the times before and after the fault occurs. Real-time fault data will be sent via mobile transmission channel immediately with the top priority once a fault is detected.

$>$ Real-time fault notification - a full description of all the major faults currently existing in a whole EMU, which ensures any major fault being sent to the ground as a complement of the real-time fault data. Fault notification will be periodically summarized and sent via mobile transmission channel at a fixed interval time, e.g. 1 minute.

$>$ Real-time operational condition data - the data that describes the key operating status of critical subsystem/device of a whole set of EMU, including traction, brake, pantograph voltage, axle temperature, air conditioning, doors, toilets, water supply, etc., mainly for operation monitoring. Real-time operation data will be collected and sent via mobile transmission channel at a fixed interval time, e.g. 1 minute.

Non-real-time train data - all the faults and operational condition data that the device can acquire from the TCMS, mainly for maintenance-oriented fault analysis and operational trend analysis. Non-real-time train data will be sent to the ground via WALN transmission channel when an EMU stops in a depot or a maintenance base where a wireless LAN is configured for the uploading connection access of the EMU.

Self-diagnosis data and event log - self-diagnosis data and event log of the transmission device itself, mainly for facilitating the operation management and maintenance of onboard train data wireless transmission devices. Selfdiagnosis data will be sent to the ground via mobile transmission channel when an EMU is powered on. The log is to keep records of the operating and data transmission events of the device and available for analysis, which can be uploaded via WALN transmission channel at a request from the ground or dumped by a portable computer for optimizing transmission procedure or analyzing any abnormality occurring to the device.

\section{Design requirements for onboard transmission device}

Aimed at improving the operation management and the maintenance of the onboard train data wireless transmission device, the design requirements are given to offer reference for design or redesign such a standard device. It was recommended that the device is mainly composed of a master machine antennas, and onboard software.

\subsection{Hardware design}

The master machine can be modularized into six parts as shown in Figure 1, including an EMU TCMS data interface unit, a train data processing unit, a mobile transmission unit for real-time data, a WLAN transmission unit for nonreal-time data, a data storage unit, and a data dump unit.

The EMU TCMS data interface unit acquires train data via a unidirectional interface with security isolation features from the Train Control and 


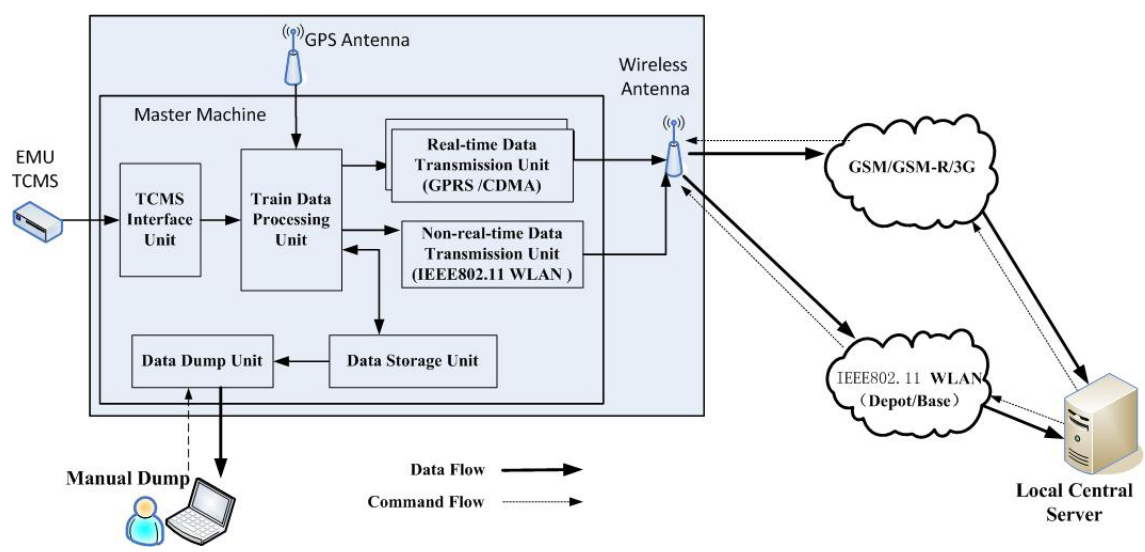

Figure 1: $\quad$ Modularization of train data wireless transmission device.

Management System. Besides, Ethernet and USB connection interfaces will be afforded for a portable PC to manually dump the train data as a last resort when the WLAN transmission unit fails.

As for the mobile transmission unit, multiple mobile data communication modes used in China will be taken into consideration and several options, e.g. GPRS, WCDMA, or CDMA-EVDO, TDSCDMA, can be available for a specific installation according the coverage and QOS of different operators' mobile communication network.

The antennas include the mobile transmission antenna for real-time data, the wireless transmission antenna for non-real-time data, and the GPS antenna for train positioning with a location precision of no less than $10 \mathrm{~m}$. These antennas can be combined in one installation or be shared with other onboard equipment depending on specific design.

Besides uploading the six categories of train data to the ground, it is recommended that commands can sent from the ground to the onboard wireless transmission devices to afford remote management of these devices, e.g., resetting the parameters of a software in the device remotely.

\subsection{Software design}

The onboard software is installed in the master machine and has the following main functions:

$>$ acquires related data from the TCMS according to the train data requirements.

$>$ converts the data acquired from the TCMS into pre-defined formats and stores it in FIFO files with specified names.

$>$ supports TCP/IP data transmission with priority control, e.g. TCP for GPRS, UDP and TCP for WLAN, over GPRS/WCDMA/CDMA-EVDO/TDSCDMA communication channel and WLAN communication channel.

$>$ completes reliable uploading of various real-time and non-real-time data based on train-ground data communication protocols. 
affords device management support functions, including self-diagnosis, logging, remote parameter configuration, data re-uploading request, etc. To normalize the functions of self-diagnosis and logging, the codes of basic selfdiagnosis contents and their related parameters are defined and the format of $\log$ files is common for all types of the EMUs.

\section{Train data wireless transmission and management system}

The normalization of train data category definition and the specification of a standard train data wireless transmission device for CRH EMUs have made it possible to set up a unified train data management system, which can receive all the uploading train data, store it in a large-scale Oracle $10 \mathrm{~g}$ database and afford simultaneous database access for various users in their desired data views supported by specific application programs.

\subsection{Generalization and standardization of train data}

Except GPS data and self-diagnosis data and event log of the transmission device itself, the specific contents of the other four categories of train data for each type of CRH EMU are still quite different due to their characteristic design, e.g., item codes, fault codes, definition of parameters, classification of faults/alarms and classification criterion, etc.

To afford unified and collective train data views to cater the needs of the users, the initial train data uploaded by the EMUs will be further handled as it is stored in normalized database tables. We adopted some approaches to generalizing and standardizing the differentiated data, e.g. additional summary fields or columns, additional summary data tables, cross-reference code tables binding unified classification codes with specific classification codes.

\subsection{Related data sources}

To cater the needs of integral data access, some related data sources must be incorporated with the train data in opportune manners. Major related data sources are:

$>$ train properties and basic configuration, crew assignment, maintenance schedule, faults recently detected during the process of servicing and maintenance of the EMUs, etc.

$>$ GIS data of all railway lines that run the CRH EMUs, to enable the conversion of GPS train position into line millage and afford map-based tracking and tracing of EMUs. This kind of data can be retrieved from the RGIS system, which stores GIS data of most Chinese railway lines, via customized services of the system.

train operation diagram, train delays, exceptional train stops, the conditions of some onboard signal equipment not integrated in the EMU TCMS, and line characteristic parameters when a exceptional train stop happens, which is mainly from the Traffic Management System and the EMU Operation Analysis System. 
fault knowledge base, including fault code, fault location, fault description, potential fault reasons, summary of fault shooting procedure, summary of fault handling process, etc., mainly abstracted from EMU's driver operation manuals and fault manuals and kept in normative database tables.

\subsection{System composition}

The composition of the train data wireless transmission and management system can be shown as Figure. 2, which can be divided into two layers, the upper layer of operation management and the lower layer of maintenance field.

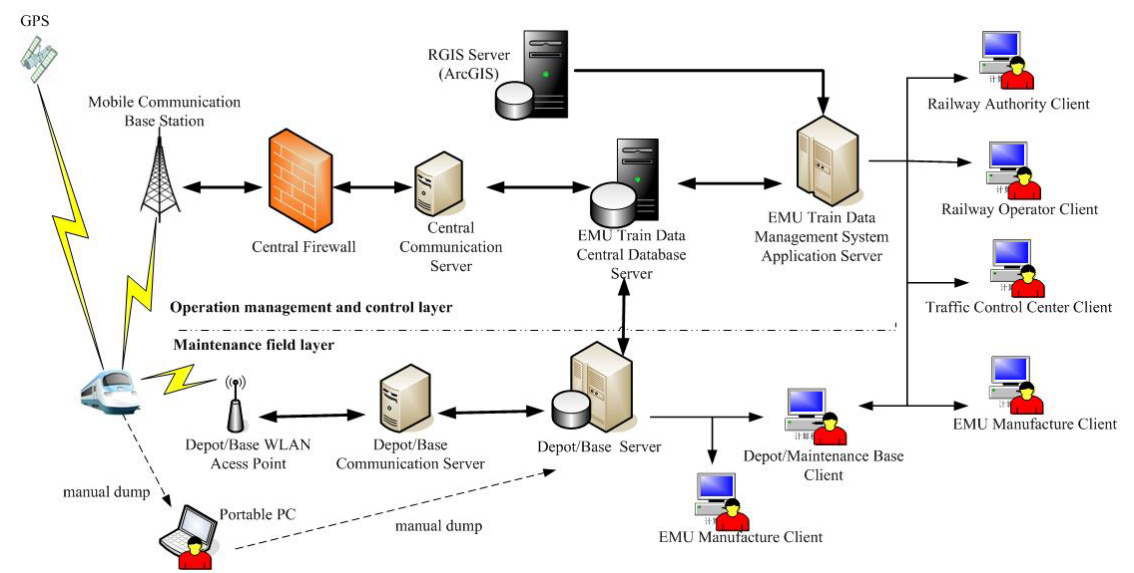

Figure 2: Composition and data flow of the unified train data wireless transmission and management system.

All real-time data is sent to store in the central database at the upper layer and can be incorporated with GIS data in the RGIS data to enable map-based monitoring and queries for various users.

Non-real-time train data is firstly uploaded via WLAN transmission channel to the servers in the EMU depots and maintenance bases at the field layer local analysis. Some summarized data, statistics data, and confirmed faults will be further uploaded to the central database at the upper layer after the initial nonreal-time train data is precessed and screened by the field technical personnels.

\subsection{Functionalities and applications}

At the present stage, the main functions of the train data management system are considered as follows:

$>$ map-based operation tracking and tracing - all the EMUs currently in operation can be displayed on a map with coloured icons indicating their current operating status, i.e., running, stopping, running with faults, stopping with faults, shown as Figure 3. For a selected EMU, its operating status in a specified period of time can also be traced in a map-like view. 


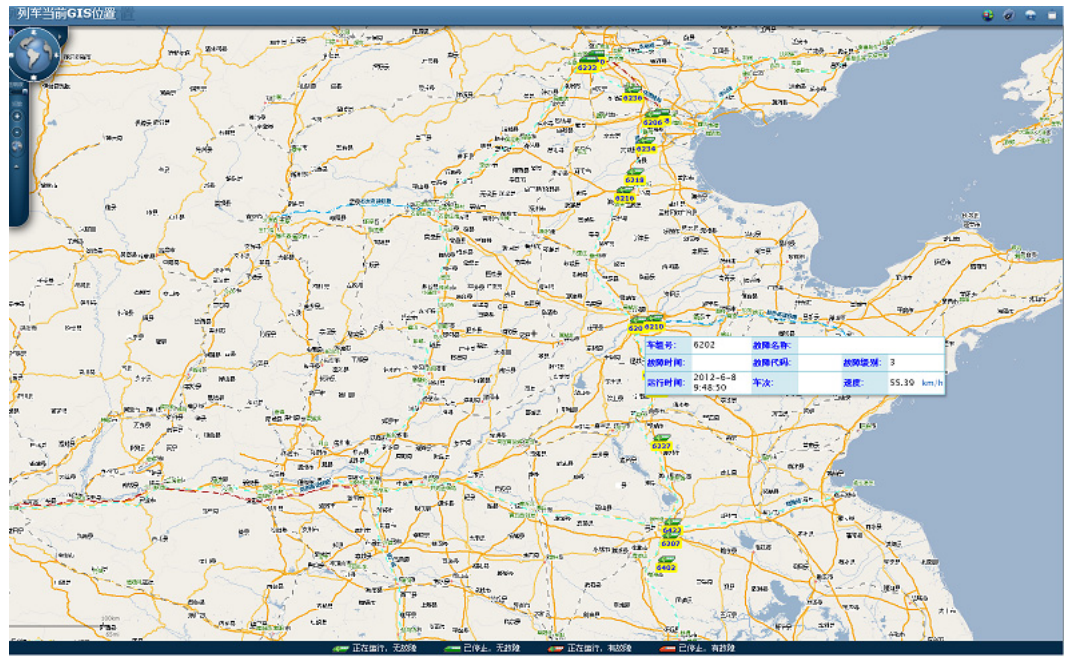

Figure 3: $\quad$ Map-based operation tracking window.

remote expert support in emergency response - real-time faults can be viewed by the remote experts in a similiar display pattern as the drivers and crew view on the onboard monitor or display screens, facilitating the communication between the experts and the crew on the EMU. Figure 4 is an example of such real-time fault notification window for $\mathrm{CRH} 2 \mathrm{C}$. Detailed fault description, fault shooting procedure, and handling instruction can be easily retrieved from the fault knowledge base of the system.

fault tracking and statistics - e.g., fault serial incident tracking for all or any subsystem of a specified EMU, summary and statistics of the faults in a specified period.

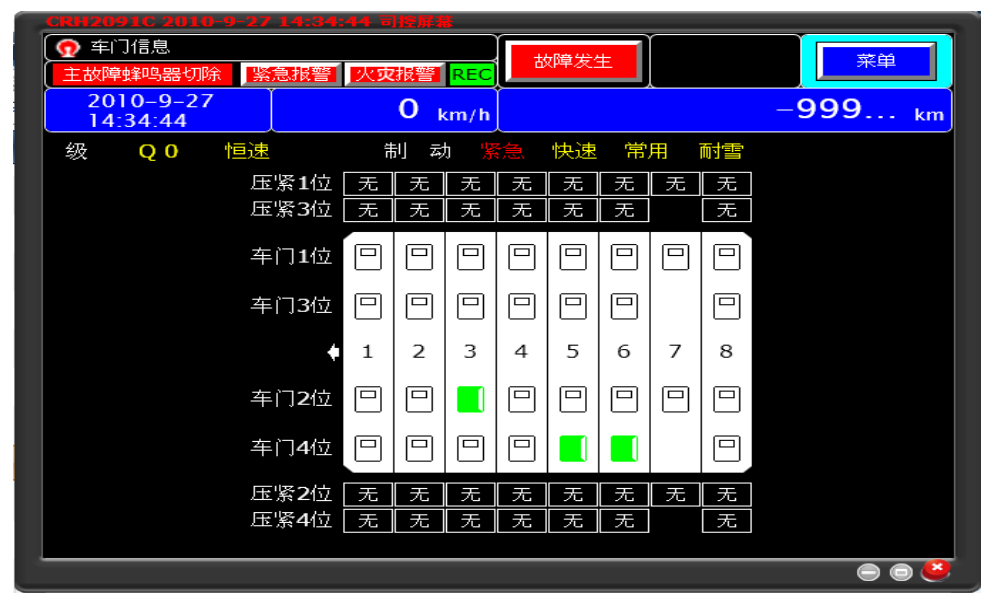

Figure 4: $\quad \mathrm{CRH} 2 \mathrm{C}$ real-time fault notification window. 
operating condition trend analysis - trend curves can be depicted using the operating parameters of any subsystema or critical components. Shown in Figure 5 is an example window of the $\mathrm{BC}$ pressure trend curve of a $\mathrm{CRH} 2 \mathrm{C}$ EMU.

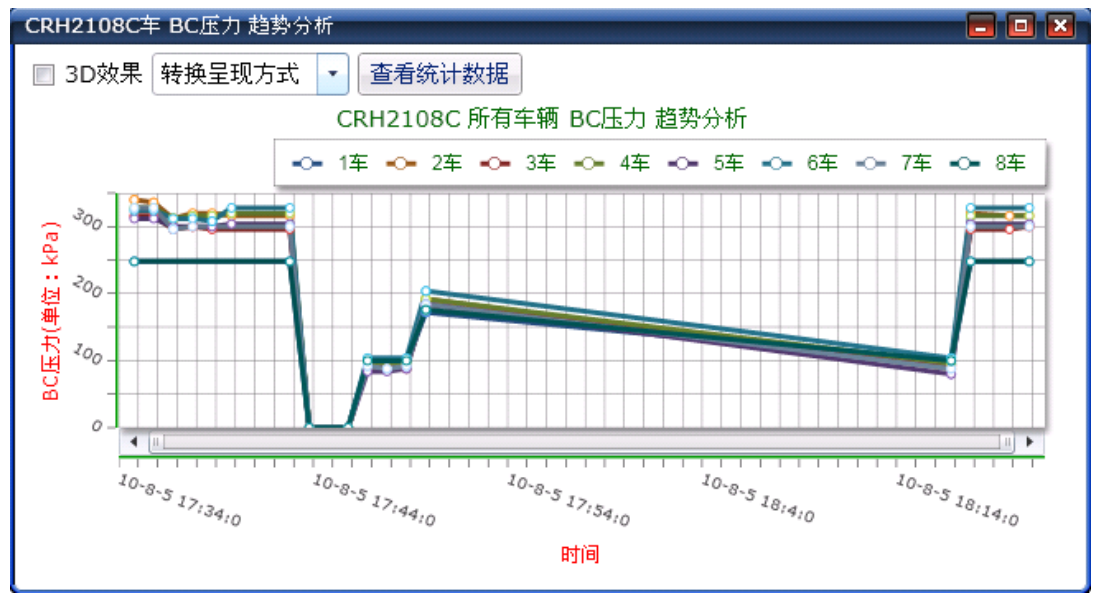

Figure 5: $\quad \mathrm{CRH} 2 \mathrm{C}$ trend curve view window $-\mathrm{BC}$ pressure.

fault repair notification and reliability monitoring - the faults automatically detected by the on-board fault diagnostic devices will be combined with the faults reported by the crews and the servicing and maintenance staff and be arranged into job orders by the dispatchers in the depots and maintenance bases. The incidences of the same faults in certain subsystems or critical components in succession will be monitored based on the tool of statistical quality control, i.e., SQC. Once such a fault is discovered, a subsequent investigation can be launched to find out the potential dominating reason and specific measures will be taken to reduce or avoid such a fault in the later.

after-sales service response and technical support - all the faults can be viewed by after-sales service personnel of the EMU manufacturers and related monitoring data can also be shared by their technical experts.

auxiliary onboard device management - the operating conditions of the entire onboard standard train data transmission device can be monitored on-line by system management facilities and a failed device will be repaired or replaced on time, minimizing the interruption of train data transmission.

\section{Progress and prospect of the project}

\subsection{Progress}

Since the year of 2011, batches of the newly developed CRH380 series EMUs and newly manufactured CRH2C series EMUs have been installed with the standard onboard transmission devices. By now, there are 71 CRH380A EMUs, 
55 CRH380B EMUs, and 30 CRH2C EMUs installed with such a transmission device. For those old types of EMUs already in service, their manufactures have redesigned and developped new type onboard transmission devices according to the specifications and are conducting device modification and field transmission trials on fews of pilot EMUs. After the trials have been validated, more old types of EMUs will be brought into the system.

Through the real-time data transmission tests conducted on the ZhengzhouXi'an PDL, the Wuhan-Guangzhou PDL, and the Beijing-Shanghai high speed railway, it was demonstrated that real-time train data could be sent to the ground at a satisfactory level of stability and real-timeness even when the train speed approached $350 \mathrm{~km} / \mathrm{h}$. Meanwhile, the WLAN-based non-real-time transmission and uploading tests had been conducted in Wuhan EMU Maintenance Base. Thorough testing and research work was focused on the communication protocols, overall arrangement and optimization of wireless coverage, manners of data storage, and policies of data transmission. The tests had validated it that manual dump can be completely replace by automatic uploading via WLAN connection.

The applications of map-based train operation monitoring, emergency response support, operating condition trend analysis, fault tracking and statistics, fault repair notification mainly based on real-time train data have been availed and more specialized maintenance-oriented analysis applications are being developed under the collaboration of the EMU manufacturers and related institutes.

\subsection{Prospect}

So far, the total mileage of high speed lines at the speed of $200 \mathrm{~km} / \mathrm{h}$ and above in China has surpassed $11,000 \mathrm{~km}$, including more than $3,000 \mathrm{~km}$ speed-raised existing railway lines. And the fleets of CRH EMUs in service currently has surpassed 700 sets and is expected to reach 880 sets by the end of this year. With the rapid expansion of the operation of CRH EMUs, keeping a safe and smooth operation of those EMUs is an arduous task full of challeges. The Chinese railways need to utilize advanced means to enhance the level of operation and maintenance management. On the basis of dynamic standardization, this project is aimed to unify the management and utilization of the train data from all types of CRH EMUs in an integrated solution, which will strengthen the long-term cooperation among the CRH EMU manufacturers and the railway operators and help improve the reliability and maintenability of CRH EMUs in the future.

\section{Acknowledgements}

Initiating the project and developing the system would have been an impossible task without the support of all the CRH EMU manufacturers. They have always taken active participation in this project and their collaborative mindset and incessant endeavors will push forward the progress of the project. 


\section{References}

[1] Technical specification of train data wireless transmission devices for CRH series EMU, Transportation Department of the Ministry of Railway, May, 2010.

[2] Requirements for filling vehicle technical data of CRH Series EMU, Transportation Department of the Ministry of Railway, March, 2011.

[3] Reports on the current situation of EMU Operation Analysis System, Locomotive Section of the Transportation Department of the Ministry of Railway, March, 2012.

[4] Testing reports on CRH380 EMU real-time train data transmission on Zhengzhou-Xi'an PDL, the Wuhan-Guangzhou PDL, and the BeijingShanghai high speed railway, Institute of Computing Technologies, CARS, 2011.

[5] Initial testing report on CRH380 EMU non-real-time train data transmission in Wuhan EMU maintenance base, Institute of Computing Technologies, CARS, 2011. 